\title{
A study to investigate the reduction in exposure to selected cigarette smoke constituents in healthy Japanese smokers who switched to a novel tobacco vapor product
}

\author{
Takeshi Kurachi, Kyoko Nakaya, Yuki Takeshige, Yasuyuki Futamura
}

Japan Tobacco Inc., Japan

\section{[Background]}

We have recently developed a novel tobacco vapor product (NTV) as an alternative to traditional tobacco products. The NTV generates a thermally-vaporized aerosol which then passes through a tobacco-containing capsule before being inhaled. In doing so, evaporated tobacco-derived flavors and nicotine are infused into the vapor. This mechanism of tobacco vapor generation differs from most existing heated tobacco products in that it does not heat the tobacco directly during use. The NTV also differs from most e-cigarettes as the carrier liquid does not contain nicotine. We have confirmed that the most measured cigarette smoke constituents are below quantifiable levels in NTV vapor. The primary objective of this clinical study was to demonstrate the reduction in exposure to selected cigarette smoke constituents, including harmful and potentially harmful constituents (HPHCs), found in tobacco products and tobacco smoke (Food and Drug Administration, 2012. Guidance for Industry - Reporting Harmful and Potentially Harmful Constituents in Tobacco Products and Tobacco Smoke under Section 904(a)(3) of the Federal Food, Drug, and Cosmetic Act - Draft Guidance).

[Methods]

This controlled clinical study randomly assigned 60 healthy Japanese smokers, aged from 21 to 65, into one of three test groups; switch to NTV use $(n=20)$, continue to smoke conventional cigarettes $(C C, n=20)$ or smoking abstinence (SA, $\mathrm{n}=20$ ) for 5 days. Fifteen biomarkers of exposure (BoEs) to selected cigarette smoke constituents, including selected HPHCs (acrolein, acrylonitrile, 4-ABP, 1-AN, 2-NA, benzene, BaP, 1,3-butadiene, CO, crotonaldehyde, ethylene oxide, NNK, NNN, o-toluidine, and pyrene), were measured. Nicotine uptake, product consumption, subjective effects, and puffing topography were also assessed during the course of the study.

[Results]

The levels of fifteen BoEs were significantly reduced in NTV group compared to the CC group and were similar to that of the SA group. Nicotine equivalents in the NTV group was intermediate between that of the CC and SA groups.

[Conclusions]

The results of this study confirmed that exposure to cigarette smoke constituents, including selected HPHCs, decreases drastically when switching to NTV use for 5 days. 